\title{
Comparison of long-term outcomes of endoscopic submucosal dissection and surgery for esophagogastric junction adenocarcinoma
}

\author{
Eun Jeong Gong ${ }^{1} \cdot$ Do Hoon Kim ${ }^{1} \cdot$ Ji Yong Ahn ${ }^{1} \cdot$ Kee Wook Jung ${ }^{1} \cdot$ \\ Jeong Hoon Lee ${ }^{1} \cdot$ Kee Don Choi ${ }^{1} \cdot \mathrm{Ho} \mathrm{June} \mathrm{Song}^{1} \cdot$ Gin Hyug Lee ${ }^{1}$. \\ Hwoon-Yong Jung ${ }^{1} \cdot$ Hee Sung Kim² ${ }^{2}$ In-Seob Lee ${ }^{2}$ Beom Su Kim² ${ }^{2}$ \\ Moon-Won $\mathrm{Yoo}^{2} \cdot$ Sung Tae $\mathrm{Oh}^{2} \cdot$ Jeong Hwan Yook ${ }^{2} \cdot$ Byung Sik Kim ${ }^{2}$
}

Received: 6 September 2016/Accepted: 1 December 2016/Published online: 19 December 2016

(C) The International Gastric Cancer Association and The Japanese Gastric Cancer Association 2016

\begin{abstract}
Background Endoscopic submucosal dissection (ESD) has become accepted as the standard treatment for early gastric cancer. However, comparative outcomes of ESD and surgery have not been evaluated for adenocarcinoma in the esophagogastric junction (EGJ). We investigated the longterm outcomes of ESD compared with those of surgery for adenocarcinoma in the EGJ.

Methods Patients who underwent ESD or surgery for Siewert type II adenocarcinoma between 2005 and 2010 and who met the absolute and expanded criteria for endoscopic resection were eligible. Clinical features and treatment outcomes were retrospectively reviewed using medical records.

Results Of the 79 patients included, 40 underwent ESD and 39 underwent surgery. During the median follow-up period of 60.9 months (range, 13.1-125.4 months), the 5-year overall survival rates were $93.9 \%$ and $97.3 \%$ for the ESD and surgery groups, respectively $(p=0.376)$. There were no gastric cancer-related deaths in either group. Adverse events occurred in 11 patients (13.9\%) overall, and the incidence of treatment-related adverse events was similar between the two groups $(10.0 \%$ vs. $17.9 \%$, $p=0.308)$.
\end{abstract}

Do Hoon Kim

dohoon.md@gmail.com

1 Department of Gastroenterology, Asan Medical Center, University of Ulsan College of Medicine, 88, Olympic-ro 43-gil, Songpa-gu, Seoul 05505, Korea

2 Department of Surgery, Asan Medical Center, University of Ulsan College of Medicine, 88, Olympic-ro 43-gil, Songpa-gu, Seoul 05505, Korea
Conclusions ESD may be an effective alternative to surgery for the treatment of early gastric cancer in the EGJ based on the comparable long-term outcomes.

Keywords Gastric neoplasms · Esophagogastric junction · Endoscopic submucosal dissection · Surgery

\section{Introduction}

Adenocarcinoma of the esophagogastric junction (EGJ) has shown an increasing trend over the last decades, particularly in Western countries [1,2]. In contrast, studies on the incidence of EGJ adenocarcinoma have found no evidence of changing trends in Korea [3, 4]. EGJ adenocarcinoma has a different etiology and clinicopathological features compared to distal gastric carcinoma. Because most adenocarcinomas of the EGJ are diagnosed at an advanced stage and with a higher incidence of lymph node metastasis, the prognosis is worse than for those involving the distal stomach [5-7]. Hence, early detection and treatment is important for patients with EGJ adenocarcinoma to improve survival. However, only a few studies have focused on the clinical course of early gastric cancer (EGC) in the EGJ $[7,8]$.

Endoscopic submucosal dissection (ESD) has been accepted as the standard treatment for EGC because of its minimal invasiveness and favorable outcomes in suitable indications [9, 10]. With effective endoscopic surveillance, gastric cancers are increasingly diagnosed at an early stage. The proportion of early-stage EGJ adenocarcinoma cases has also shown an increasing trend over time, which may lead to more favorable clinical outcomes [4]. Given that the surgical treatment of EGJ carcinoma requires total gastrectomy, which has a negative impact on 


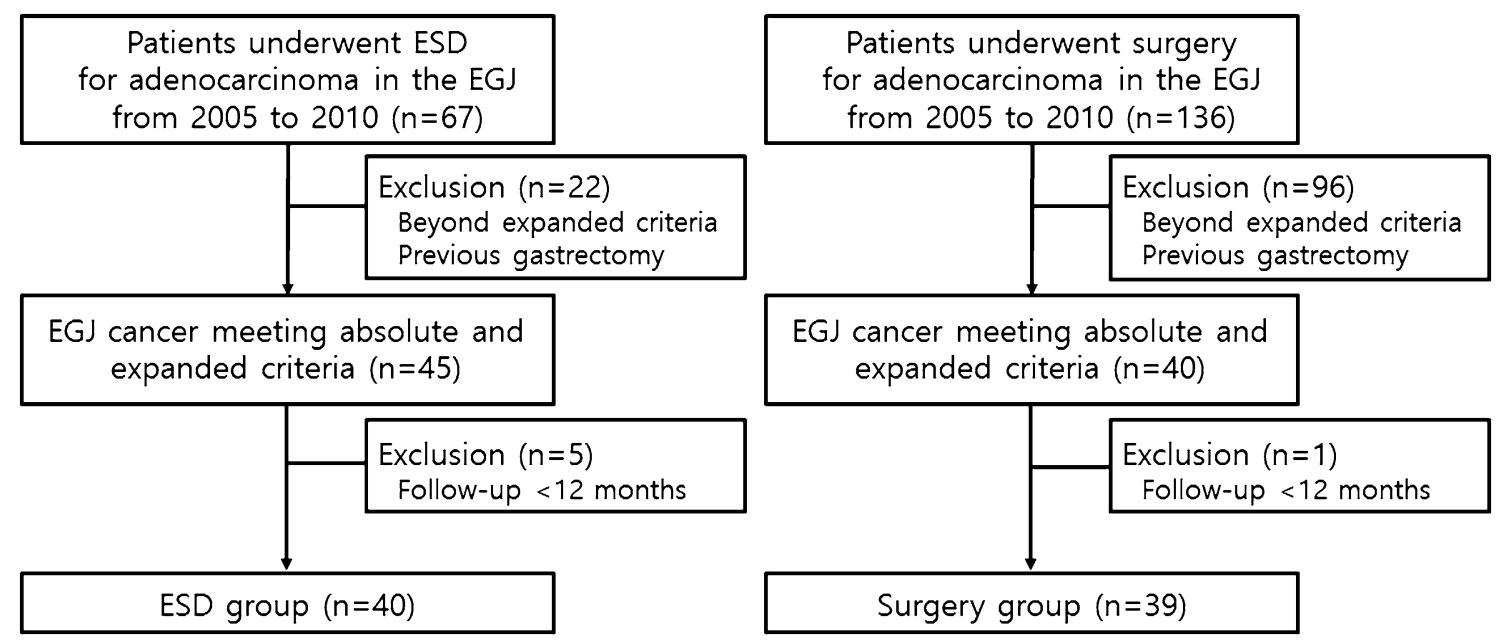

Fig. 1 Flowchart of the study. EGJ esophagogastric junction; ESD endoscopic submucosal dissection

the patient's quality of life, it seems worthwhile to investigate the clinical outcomes of ESD and assess the advantages of ESD over surgery for EGC in the EGJ separately. Although there have been several studies reporting the clinical outcomes of ESD for EGJ adenocarcinoma, comparative outcomes of ESD and surgery have not been evaluated [11-15]. Therefore, we compared the clinical outcomes of ESD with those of surgery for EGJ adenocarcinoma using long-term follow-up data.

\section{Methods}

\section{Patients}

Patients who underwent ESD or surgery for Siewert type II adenocarcinoma between January 2005 and December 2010 and who met the absolute and expanded criteria for endoscopic resection were eligible. The diagnosis of EGJ cancer was based on endoscopic findings and gross findings of resected specimens, with the distal limit of palisading vessels or the proximal limit of the gastric rugal folds as landmarks for the EGJ. Siewert type II adenocarcinoma is defined as a tumor with an epicenter located within $1 \mathrm{~cm}$ proximal and $2 \mathrm{~cm}$ distal to the EGJ. The clinicopathological characteristics and treatment outcomes were retrospectively reviewed using medical records. This study was approved by the Asan Medical Center Institutional Review Board.

During the study period, a total of 203 patients diagnosed with EGJ adenocarcinoma were treated with ESD $(n=67)$ or surgery $(n=136)$ at Asan Medical Center. Of these, 11 patients who had previously undergone gastric surgery and 107 who did not meet the absolute or expanded criteria for endoscopic resection were excluded. In addition, 6 patients whose follow-up period after treatment was less than 12 months were excluded. Ultimately, 79 patients were analyzed. A detailed flowchart of the study is shown in Fig. 1.

\section{Treatment and follow-up}

The choice of treatment was based on clinical information, the patient's medical condition, and patient preferences after explaining the advantages and disadvantages of each treatment modality. Endoscopic resection was performed by one of six experienced endoscopists, with all patients under conscious sedation. The detailed ESD technique has been described previously [16]. Patients treated by surgery underwent an open or laparoscopic total gastrectomy with lymph node dissection, with or without esophagectomy. Follow-up endoscopy was performed every 6-12 months during the first 2 years after treatment and annually thereafter. A computed tomography scan of the abdomen was performed annually.

\section{Definitions}

Patients were classified according to absolute and expanded criteria based on the final pathological diagnosis [17]. The absolute indication for endoscopic resection was mucosal, differentiated tumor without ulceration, $\leq 2 \mathrm{~cm}$. The expanded indications for EGC comprised the following criteria: (1) mucosal, histologically differentiated tumor without ulcerative findings, $>2 \mathrm{~cm}$; (2) mucosal, histologically differentiated tumor with ulceration, $\leq 30 \mathrm{~mm}$; and (3) minute submucosal tumor $(<500 \mu \mathrm{m}$ from the muscularis mucosae), histologically differentiated 
type, $\leq 30 \mathrm{~mm}$. Histologically differentiated types included well or moderately differentiated tubular adenocarcinoma and papillary adenocarcinoma. Undifferentiated types included poorly differentiated tubular adenocarcinoma, signet ring cell carcinoma, and mucinous adenocarcinoma [18].

The macroscopic types of tumor were defined according to the Japanese classification of gastric carcinoma [18]: type I (protruded), type IIa (superficial elevated), type IIb (flat), type IIc (superficial depressed), and type III (excavated). Types I and IIa were classified as elevated types, types IIc and III as depressed types, and type IIb as a flat type.

En bloc resection was defined as resection of lesions in one piece regardless of the depth of invasion or lymphovascular invasion. Complete resection was defined as resection of the tumor with horizontal and vertical margins free of tumor. Even when tumors were resected in piecemeal fashion, complete resection was defined as removal of the entire lesion including tumorfree margins after reconstruction of all pieces. Resection was considered curative when en bloc complete resection was achieved and the tumor met the absolute or expanded criteria in the absence of lymphovascular invasion. When tumors did not fulfill the foregoing criteria for curative resection, resection was considered noncurative. Tumors detected at the resection site were defined as locally recurrent tumors. Tumors detected at sites other than the primary resection site within 12 months were regarded as synchronous tumors, whereas tumors detected more than 12 months after ESD were regarded as metachronous.

Adverse events occurring within 30 days of treatment were defined as early adverse events and those occurring thereafter as late adverse events. Adverse events were classified according to the Clavien-Dindo classification [19]. Stricture was defined as gross narrowing of the EGJ or anastomosis site and the presence of symptoms related to the obstruction or when resistance was felt when the endoscope was passed through.

\section{Statistical analysis}

Differences between clinical characteristics were assessed using the chi-square test, Fisher's exact test, or the Mann-Whitney $U$ test, as appropriate. Overall and disease-specific survival rates, and cumulative recurrence rates, were analyzed by the Kaplan-Meier method, and comparisons were performed using the log-rank test. All statistical analyses were performed using SPSS 21.0 (SPSS, Chicago, IL, USA), and a $p$ value $<0.05$ was considered statistically significant.

\section{Results}

\section{Baseline and clinicopathological characteristics}

Of the 79 patients included in the study, 40 underwent ESD (ESD group) and 39 underwent surgery (surgery group). Baseline and clinicopathological characteristics are summarized in Tables 1 and 2. The median age of the 79 patients was 64 years (range, 40-80 years), and the maleto-female ratio was 7.8:1. Comorbidities including diabetes mellitus, cardiovascular disease, coronary artery disease, liver cirrhosis, chronic pulmonary disease, end-stage renal disease, and cancer were present in 27 patients (34.2\%). The median tumor size was $21.0 \mathrm{~mm}$ (interquartile range, $14.0-30.0 \mathrm{~mm})$. Of all the tumors, $46(58.2 \%)$ were well differentiated and $33(41.8 \%)$ moderately differentiated adenocarcinomas. Sixty-four lesions $(81.0 \%)$ were confined to the mucosal layer, whereas $15(19.0 \%)$ invaded the submucosal layer. According to the final pathological diagnosis, 29 lesions (36.7\%) were included in the absolute criteria group and $50(63.3 \%)$ in the expanded criteria group. The median number of lymph nodes that were resected during surgery was 33 (interquartile range, 27-37), and none of these revealed metastasis. Patients in the ESD group were younger, had significantly smaller tumors, and had fewer moderately differentiated adenocarcinomas and submucosal cancers than those in the surgery group.

\section{Clinical outcomes of ESD}

The clinical outcomes of ESD are shown in Table 3. Of the 40 patients who underwent ESD, en bloc resection was achieved in 37 lesions (92.5\%), with complete resection for 35 lesions $(87.5 \%)$ and curative resection for 33 lesions $(82.5 \%)$. Of the 7 patients with noncurative resection, 2 underwent subsequent surgery, and none of these patients had lymph node metastases. The remaining patients were followed up by endoscopy, either because of advanced age $(n=1)$, the cause of noncurative resection being solely that of piecemeal resection $(n=3)$, or involvement of the horizontal resection margin by adenoma $(n=1)$. None of these patients developed local recurrence.

\section{Comparison of long-term outcomes of ESD and surgery}

During the median follow-up period of 60.9 months (range, 13.1-125.4 months), the 5-year overall survival rates were 93.9\% and $97.3 \%$ for the ESD and surgery groups, respectively ( $p=0.376$ ) (Fig. 2). Because there were no gastric cancer-related deaths during follow-up, the 5-year 
Table 1 Baseline characteristics of the study population

\begin{tabular}{lllr}
\hline Factor & ESD $(n=40)$ & Surgery $(n=39)$ & $p$ value \\
\hline Age, years & $65(61-69)$ & $60(57-68)$ & 0.041 \\
Male sex & $35(87.5)$ & $35(89.7)$ & $>0.999$ \\
Comorbidity & & & \\
Diabetes mellitus & $3(7.5)$ & $7(17.9)$ & 0.193 \\
Cardiovascular disease & 0 & $2(5.1)$ & 0.241 \\
Coronary artery disease & $3(7.5)$ & $2(5.1)$ & $>0.999$ \\
Liver cirrhosis & $1(2.5)$ & $1(2.6)$ & $>0.999$ \\
Chronic pulmonary disease & $3(7.5)$ & 0 & 0.241 \\
End-stage renal disease & 0 & $1(2.6)$ & 0.494 \\
Malignancy & $4(10.0)^{\mathrm{a}}$ & $3(7.7)^{\mathrm{b}}$ & $>0.999$ \\
Helicobacter pylori infection & $23(57.5)$ & $4(36.4)^{\mathrm{c}}$ & 0.214 \\
Family history of gastric cancer & $9(22.5)$ & $7(17.9)$ & 0.781 \\
Smoking & & $20(51.3)$ & 0.175 \\
Never & $13(32.5)$ & $9(23.1)$ & \\
Previous & $16(40.0)$ & $10(25.6)$ & \\
Current & $11(27.5)$ & $14(35.9)$ & 0.939 \\
Alcohol consumption & & $3(7.7)$ & \\
Never & $16(40.0)$ & $22(56.4)$ & \\
Previous & $21(52.5)$ & & \\
Current & & &
\end{tabular}

Data represent the number of patients $(\%)$ or the median (interquartile range)

ESD endoscopic submucosal dissection

${ }^{a}$ Two patients had histories of cancer (hepatocellular carcinoma and colon cancer), and two patients underwent surgery for double primary colon cancer simultaneously at the time of total gastrectomy

b One patient had a previous history of colon cancer, and two patients underwent surgery for double primary cancer (renal cell carcinoma and colon cancer) simultaneously at the time of total gastrectomy

${ }^{c}$ Helicobacter pylori infection status was assessed in 11 of the 39 patients disease-specific survival rate was $100 \%$ in both groups. There were no local recurrences of gastric cancer after ESD or surgery. Synchronous or metachronous lesions developed in four patients in the ESD group. All four underwent endoscopic resection, and curative resection was achieved in three cases; the remaining patient had deep submucosal invasion after endoscopic resection.

\section{Adverse events associated with treatment}

Adverse events occurred in 11 patients (13.9\%) (Table 4). The incidence of overall treatment-related adverse events was similar in the two groups $(10.0 \%$ vs. $17.9 \%$; $p=0.308)$. Of the adverse events, 7 were Clavien-Dindo grade III or higher [3 $(7.5 \%)$ in the ESD group and 4 $(10.3 \%)$ in the surgery group; $p=0.712]$. In the ESD group, 1 patient required emergency surgery because of uncontrolled bleeding during the procedure. There were two cases of exposure and tearing of the muscularis propria layer during ESD; these were managed by endoclipping and supportive care. Pneumoperitoneum was not evident by radiography after the procedure, and these patients were discharged without any further intervention. In the surgery group, 1 patient underwent surgical management for mechanical ileus caused by internal herniation that occurred a month after surgery. Another patient had complicated fluid collection in the surgical bed and was treated with repeated percutaneous drainage and intravenous antibiotics. Duration of hospital stay was significantly shorter in the ESD group than in the surgery group (median, 4 vs. 9 days; $p<0.001$ ). There were no treatment-related mortalities in either group.

\section{Discussion}

It is thought that adenocarcinomas of the EGJ have different clinicopathological characteristics and are associated with worse survival than distal gastric cancer, which has a 5 -year overall survival rate of $90.6 \%$ for surgically resected pT1 cancer [5-8]. Therefore, we investigated the long-term outcomes of EGC in the EGJ separately, and found that the 5-year overall and disease-specific survival rates after ESD were comparable to those after surgery. The incidence of 
Table 2 Clinicopathological features of adenocarcinoma in the esophagogastric junction

\begin{tabular}{|c|c|c|c|}
\hline & $\operatorname{ESD}(n=40)$ & Surgery $(n=39)$ & $p$ value \\
\hline Size of tumors $(\mathrm{mm})$ & $17.5(13.0-24.8)$ & $24.0(16.0-35.0)$ & 0.008 \\
\hline Histological differentiation & & & 0.032 \\
\hline Well differentiated & $28(70.0)$ & $18(46.2)$ & \\
\hline Moderately differentiated & $12(30.0)$ & $21(53.8)$ & \\
\hline Barrett cancer & $4(10.0)$ & 0 & NA \\
\hline Macroscopic type & & & 0.545 \\
\hline Elevated & $15(37.5)$ & $11(28.2)$ & \\
\hline Flat & $13(32.5)$ & $12(30.8)$ & \\
\hline Depressed & $12(30.0)$ & $16(41.0)$ & \\
\hline Depth of invasion & & & 0.039 \\
\hline Mucosa & $36(90.0)$ & $28(71.8)$ & \\
\hline Submucosa & $4(10.0)$ & $11(28.2)$ & \\
\hline Lymphovascular invasion & 0 & $1(2.6)$ & NA \\
\hline Perineural invasion & 0 & 0 & NA \\
\hline Criteria for endoscopic resection & & & 0.001 \\
\hline Absolute criteria & $22(55.0)$ & $7(17.9)$ & \\
\hline Expanded criteria & $18(45.0)$ & $32(82.1)$ & \\
\hline Follow-up (months) & $63.2(53.6-83.5)$ & $60.3(58.4-68.7)$ & 0.860 \\
\hline
\end{tabular}

Data represent number of patients (\%) or median (interquartile range)

$E S D$ endoscopic submucosal dissection, $N A$ not assessed
Table 3 Clinical outcomes of endoscopic resection

\begin{tabular}{ll}
\hline Criteria for endoscopic resection & $22(55.0)$ \\
Absolute criteria & $18(45.0)$ \\
Expanded criteria & $17.5(13.0-24.8)$ \\
Size of tumors (mm) & \\
Result of resection & $37(92.5)$ \\
En bloc resection & $35(87.5)$ \\
Complete resection & $33(82.5)$ \\
Curative resection & \\
Adverse events & \\
Bleeding & $1(2.5)$ \\
Immediate & $1(2.5)$ \\
Early delayed & 0 \\
Late delayed & $2(5.0)$ \\
Microperforation & 0 \\
Stricture & \\
Clinical outcome & \\
Local recurrence & 0 \\
Synchronous lesions & $1(2.5)$ \\
Metachronous lesions & $3(7.5)$ \\
\hline
\end{tabular}

Data represent number of patients (\%) or median (interquartile range)

treatment-related adverse events was similar in the two groups, and the length of hospital stay was shorter in the ESD group. To the best of our knowledge, the current study is the first to compare the clinical outcomes of ESD and surgery for EGJ adenocarcinoma over a substantial followup period.

Surgical resection is the mainstay of treatment for gastric carcinoma that can achieve complete removal of tumors and lymph nodes. ESD has been considered a feasible alternative to surgical resection for tumors with mucosal or minute submucosal invasion, because for these tumors there is little or no risk of lymph node metastasis $[17,20]$. Several recent studies have compared the clinical and oncological outcomes of ESD with gastrectomy, demonstrating favorable outcomes of ESD in terms of survival as well as perioperative outcomes [10, 21-23]. The long-term outcomes of ESD were comparable to that of gastrectomy, even when the analysis was restricted to EGC that met the expanded criteria [10]. In our present study, we compared the long-term outcomes of ESD to surgery for EGC in the EGJ that met the absolute or expanded criteria. The 5-year overall survival rates were $93.9 \%$ and $97.3 \%$ for the ESD and surgery groups, respectively, and the disease-specific survival rate was $100 \%$ in both groups. These results indicate that the clinical outcomes of adenocarcinomas in the EGJ are favorable when they are detected and managed at an early stage.

Although ESD has been found acceptable for the treatment of EGC, the curative resection rate for EGJ adenocarcinoma has been reported to be relatively lower than that of distal stomach $[9,24]$. This finding is thought to be due to difficulty in predicting depth of invasion and in 
Fig. 2 Comparison of the overall survival of patients with esophagogastric junction adenocarcinoma in the endoscopic submucosal dissection and surgery groups. No number, ESD endoscopic submucosal dissection

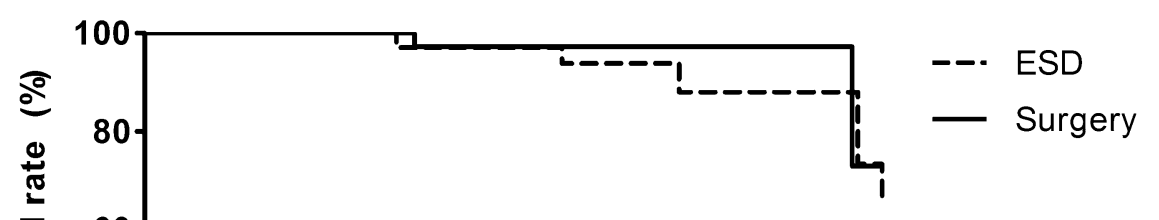

No at risk

$\begin{array}{llllllllll}\text { ESD } & 40 & 40 & 36 & 34 & 31 & 24 & 14 & 9 & 2 \\ \text { Surgery } & 39 & 39 & 37 & 36 & 35 & 21 & 8 & 4 & 2\end{array}$

Table 4 Adverse events associated with treatment

\begin{tabular}{|c|c|c|c|}
\hline & $\begin{array}{l}\text { ESD } \\
(n=40)\end{array}$ & $\begin{array}{l}\text { Surgery } \\
(n=39)\end{array}$ & $p$ value \\
\hline \multicolumn{4}{|l|}{ Early adverse events } \\
\hline Bleeding & $2(5.0)$ & $1(2.6)$ & \\
\hline Microperforation & $2(5.0)$ & 0 & \\
\hline Wound complications & 0 & $2(5.1)$ & \\
\hline Ileus & 0 & $1(2.6)$ & \\
\hline Intraabdominal fluid collection & 0 & $2(5.1)$ & \\
\hline Total & $4(10.0)$ & $6(15.4)$ & 0.518 \\
\hline \multicolumn{4}{|l|}{ Clavien-Dindo classification } \\
\hline I & 0 & 0 & \\
\hline II & $1(2.5)$ & $3(7.7)$ & \\
\hline IIIa & $2(5.0)$ & $3(7.7)$ & \\
\hline IIIb & $1(2.5)$ & 0 & \\
\hline IV & 0 & 0 & \\
\hline $\mathrm{V}$ & 0 & 0 & \\
\hline \multicolumn{4}{|l|}{ Late adverse events } \\
\hline Stricture & 0 & 0 & \\
\hline Bowel obstruction & 0 & $1(2.6)$ & \\
\hline Intraabdominal abscess & 0 & 0 & \\
\hline Wound dehiscence & 0 & 0 & \\
\hline Total & 0 & $1(2.6)$ & 0.494 \\
\hline \multicolumn{4}{|l|}{ Clavien-Dindo classification } \\
\hline I & 0 & 0 & \\
\hline II & 0 & 0 & \\
\hline IIIa & 0 & 0 & \\
\hline IIIb & 0 & $1(2.6)$ & \\
\hline IV & 0 & 0 & \\
\hline $\mathrm{V}$ & 0 & 0 & \\
\hline
\end{tabular}

Data represent number of patients $(\%)$

$E S D$ endoscopic submucosal dissection technical aspects related to the anatomical characteristics of EGJ, namely, the narrow and curved operative space. In the current study, the curative resection rate for the ESD group was $82.5 \%$ overall, $86.4 \%$ for tumors meeting the absolute criteria and $77.8 \%$ for those meeting the expanded criteria, which is comparable to those rates in previous studies on EGJ adenocarcinoma [13-16]. A positive lateral margin was the main cause of noncurative resection, and there was no local recurrence during follow-up even when no additional treatment was performed, supporting the curability of ESD.

The incidence of metachronous lesions is one of the concerns after endoscopic resection as almost all the gastric mucosa is preserved. However, considering that total gastrectomy has a significantly negative impact on patient quality of life, the preservation of gastric function might outweigh the possible disease burden of metachronous recurrence [25]. Moreover, with scheduled surveillance endoscopy, metachronous lesions can be detected at an early stage and so can be suitable candidates for endoscopic resection [26]. In our study, metachronous lesions were detected in $7.5 \%$ of patients after ESD. The interval between ESD and the detection of metachronous lesions ranged from 25.6 to 70.2 months, and all except one of these patients were treated by endoscopic resection with curative intent. As the cumulative incidence of metachronous lesions increases continuously even after several years of the index endoscopic resection, endoscopic surveillance after ESD should be scheduled for a substantial period [27]. In addition, eradication therapy for Helicobacter pylori infection has been reported to be beneficial in reducing the occurrence of metachronous gastric carcinoma after endoscopic resection [28]. 
Treatment-related adverse events may affect patient quality of life and prolong hospital stay. Previous reports on adverse events after endoscopic resection and surgery have been inconsistent. Although some reported that adverse events occurred less frequently in the ESD group than the surgery group, others found no difference between the two groups [10, 22, 23]. In our study, treatment-related adverse event rates were similar between the two groups. However, the late adverse events that required surgical management occurred only in the surgery group, which is consistent with a previous study [10]. In addition, the duration of hospital stay was significantly shorter in the ESD group than the surgery group. These results suggest that ESD is an acceptable treatment option for EGC in the EGJ.

Our study had several limitations of note. First, because our analysis was retrospective, nonrandomized, and conducted at a single center, potential selection bias and referral bias cannot be excluded. Another possible limitation is that the number of patients included in each group was small because of the relatively low incidence of cardia cancer in Korea [3, 4]. Although none of the patients in the surgery group had lymph node metastasis in this study, the possibility of lymph node metastasis cannot be completely excluded because of the limited sample size. Despite these limitations, our study compared clinical outcomes of ESD and surgery over a long follow-up period, supporting the feasibility and curability of ESD for EGJ adenocarcinoma. Our results suggest that there is a possibility of avoiding total gastrectomy in patients with EGC in the EGJ, which is of great value to patients.

In conclusion, ESD is an effective and safe treatment for EGC in the EGJ, on the basis of comparable long-term outcomes to surgery. For tumors that meet the absolute or expanded criteria for endoscopic resection, ESD is an acceptable alternative to surgery and favorable outcomes can be expected.

\section{Compliance with ethical standards}

Conflict of interest The authors declare no potential conflicts of interest.

Ethical statement All procedures followed were in accordance with the ethical standards of the responsible committee on human experimentation (institutional and national) and with the Helsinki Declaration of 1964 and later versions. The exemption from the informed consent requirement is permitted by the Asan Medical Center Institutional Review Board.

\section{References}

1. Buas MF, Vaughan TL. Epidemiology and risk factors for gastroesophageal junction tumors: understanding the rising incidence of this disease. Semin Radiat Oncol. 2013;23:3-9.
2. Marsman WA, Tytgat GN, ten Kate FJ, van Lanschot JJ. Differences and similarities of adenocarcinomas of the esophagus and esophagogastric junction. J Surg Oncol. 2005;92:160-8.

3. Lee JY, Kim HY, Kim KH, Jang HJ, Kim JB, Lee JH, et al. No changing trends in incidence of gastric cardia cancer in Korea. J Korean Med Sci. 2003;18:53-7.

4. Chung JW, Lee GH, Choi KS, Kim DH, Jung KW, Song HJ, et al. Unchanging trend of esophagogastric junction adenocarcinoma in Korea: experience at a single institution based on Siewert's classification. Dis Esophagus. 2009;22:676-81.

5. Maeda H, Okabayashi T, Nishimori I, Sugimoto T, Namikawa T, Dabanaka K, et al. Clinicopathologic features of adenocarcinoma at the gastric cardia: is it different from distal cancer of the stomach? J Am Coll Surg. 2008;206:306-10.

6. Kawaguchi T, Komatsu S, Ichikawa D, Kubota T, Okamoto K, Shiozaki A, et al. Comparison of prognostic compatibility between seventh AJCC/TNM of the esophagus and 14th JCGC staging systems in Siewert type II adenocarcinoma. Anticancer Res. 2013;33:3461-5.

7. Liu K, Zhang W, Chen X, Chen X, Yang K, Zhang B, et al. Comparison on clinicopathological features and prognosis between esophagogastric junctional adenocarcinoma (Siewert II/ III types) and distal gastric adenocarcinoma: retrospective cohort study, a single institution, high-volume experience in China. Medicine (Baltimore). 2015;94:e1386.

8. Matsuda T, Kurokawa Y, Yoshikawa T, Kishi K, Misawa K, Ohi $\mathrm{M}$, et al. Clinicopathological characteristics and prognostic factors of patients with Siewert type II esophagogastric junction carcinoma: a retrospective multicenter study. World J Surg. 2016;40:1672-9.

9. Ahn JY, Jung HY, Choi KD, Choi JY, Kim MY, Lee JH, et al. Endoscopic and oncologic outcomes after endoscopic resection for early gastric cancer: 1370 cases of absolute and extended indications. Gastrointest Endosc. 2011;74:485-93.

10. Kim YI, Kim YW, Choi IJ, Kim CG, Lee JY, Cho SJ, et al. Longterm survival after endoscopic resection versus surgery in early gastric cancers. Endoscopy. 2015;47:293-301.

11. Imai K, Kakushima N, Tanaka M, Takizawa K, Matsubayashi H, Hotta K, et al. Validation of the application of the Japanese curative criteria for superficial adenocarcinoma at the esophagogastric junction treated by endoscopic submucosal dissection: a long-term analysis. Surg Endosc. 2013;27:2436-45.

12. Yamada M, Oda I, Nonaka S, Suzuki H, Yoshinaga S, Taniguchi $\mathrm{H}$, et al. Long-term outcome of endoscopic resection of superficial adenocarcinoma of the esophagogastric junction. Endoscopy. 2013;45:992-6.

13. Omae M, Fujisaki J, Horiuchi Y, Yoshizawa N, Matsuo Y, Kubota M, et al. Safety, efficacy, and long-term outcomes for endoscopic submucosal dissection of early esophagogastric junction cancer. Gastric Cancer. 2013;16:147-54.

14. Hirasawa K, Kokawa A, Oka H, Yahara S, Sasaki T, Nozawa A, et al. Superficial adenocarcinoma of the esophagogastric junction: long-term results of endoscopic submucosal dissection. Gastrointest Endosc. 2010;72:960-6.

15. Hoteya S, Matsui A, Iizuka T, Kikuchi D, Yamada A, Yamashita $\mathrm{S}$, et al. Comparison of the clinicopathological characteristics and results of endoscopic submucosal dissection for esophagogastric junction and non-junctional cancers. Digestion. 2013;87:29-33.

16. Gong EJ, Kim DH, So H, Ahn JY, Jung KW, Lee JH, et al. Clinical outcomes of endoscopic submucosal dissection for adenocarcinoma of the esophagogastric junction. Dig Dis Sci. 2016;61:2666-73.

17. Japanese Gastric Cancer Association. Japanese gastric cancer treatment guidelines 2014 (ver. 4). Gastric Cancer. 2016. doi:10. 1007/s10120-016-0622-4. 
18. Japanese Gastric Cancer Association. Japanese classification of gastric carcinoma: 3rd English edition. Gastric Cancer. 2011;14:101-12.

19. Dindo D, Demartines N, Clavien PA. Classification of surgical complications: a new proposal with evaluation in a cohort of 6336 patients and results of a survey. Ann Surg. 2004;240:205-13.

20. Gotoda T, Yanagisawa A, Sasako M, Ono H, Nakanishi Y, Shimoda $\mathrm{T}$, et al. Incidence of lymph node metastasis from early gastric cancer: estimation with a large number of cases at two large centers. Gastric Cancer. 2000;3:219-25.

21. Chiu PW, Teoh AY, To KF, Wong SK, Liu SY, Lam CC, et al. Endoscopic submucosal dissection (ESD) compared with gastrectomy for treatment of early gastric neoplasia: a retrospective cohort study. Surg Endosc. 2012;26:3584-91.

22. Choi IJ, Lee JH, Kim YI, Kim CG, Cho SJ, Lee JY, et al. Longterm outcome comparison of endoscopic resection and surgery in early gastric cancer meeting the absolute indication for endoscopic resection. Gastrointest Endosc. 2015;81(333-41):e1.

23. Pyo JH, Lee H, Min BH, Lee JH, Choi MG, Lee JH, et al. Longterm outcome of endoscopic resection vs. surgery for early gastric cancer: a non-inferiority-matched cohort study. Am J Gastroenterol. 2016;111:240-9.

24. Lee H, Yun WK, Min BH, Lee JH, Rhee PL, Kim KM, et al. A feasibility study on the expanded indication for endoscopic submucosal dissection of early gastric cancer. Surg Endosc. 2011;25:1985-93.

25. Lee SS, Chung HY, Kwon OK, Yu W. Long-term quality of life after distal subtotal and total gastrectomy: symptom- and behavior-oriented consequences. Ann Surg. 2016;263:738-44.

26. Hahn KY, Park JC, Kim EH, Shin S, Park CH, Chung H, et al. Incidence and impact of scheduled endoscopic surveillance on recurrence after curative endoscopic resection for early gastric cancer. Gastrointest Endosc. 2016;84(4):628-638 e1.

27. Min BH, Kim ER, Kim KM, Park CK, Lee JH, Rhee PL, et al. Surveillance strategy based on the incidence and patterns of recurrence after curative endoscopic submucosal dissection for early gastric cancer. Endoscopy. 2015;47:784-93.

28. Bae SE, Jung HY, Kang J, Park YS, Baek S, Jung JH, et al. Effect of Helicobacter pylori eradication on metachronous recurrence after endoscopic resection of gastric neoplasm. Am J Gastroenterol. 2014;109:60-7. 